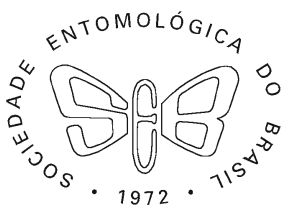

\title{
Investigations on the Genus Rhizoecus (Hemiptera: Rhizoecidae) with Description of Two New Species from South America
}

\author{
MB Kaydan ${ }^{1,2,3}$, Z Konczné Benedicty ${ }^{1}$, T Kondo $^{4}$, AA Ramos-Portilla ${ }^{5}$, É Szita ${ }^{1}$ \\ ${ }^{1}$ Plant Protection Institute, Centre for Agricultural Research, Hungarian Academy of Sciences, Budapest, Hungary \\ ${ }^{2}$ Imamoglu Vocational School, Çukurova University, Adana, Turkey \\ ${ }^{3}$ Biotechnology Research Centre, Çukurova University, Adana, Turkey \\ ${ }^{4}$ Corporación Colombiana de Investigación Agropecuaria (AGROSAVIA), Centro de Investigación Palmira, Palmira, Valle, Colombia \\ ${ }^{5}$ Instituto Colombiano Agropecuario ICA, Grupo Sistemática de Insectos Agronomía, Museo Entomológico UNAB, Facultad de Agronomía, \\ Universidad Nacional de Colombia, Bogotá, Colombia
}

\section{Keywords}

Hypogeic mealybugs, identification key, Rhizoecus, South America, taxonomy

\section{Correspondence}

É Szita, Plant Protection Institute, Centre for Agricultural Research, Hungarian Academy of Sciences, H-1022, Herman Ottó út 15, Budapest, Hungary; szita.eva@agrar.mta.hu

Edited by Douglas Zeppelini - UEPB

Received 24 January 2019 and accepted 17 March 2019

Published online: 25 April 2019

(C) The Author(s) 2019

\begin{abstract}
Neotropical species of the scale insect genus Rhizoecus Künckel d'Herculais (Hemiptera: Coccomorpha: Rhizoecidae) found in soil sample material of the Hungarian Natural History Museum were studied. Two new Rhizoecus species, Rhizoecus kontschani Kaydan and Konczné Benedicty sp. n., and Rhizoecus granaradewillinkae Kaydan and Szita sp. n., are described and illustrated based on the adult females. Also, the adult females of Rhizoecus keysensis Hambleton and Rhizoecus pseudocacticans Hambleton are illustrated. An identification key and new additional locality records for the currently known Rhizoecus species are provided.
\end{abstract}

\section{Introduction}

The rhizoecine mealybugs, formerly included in the Pseudococcidae, were elevated to family status, i.e., the Rhizoecidae (Hemiptera: Coccomorpha) by Hodgson (2012). The Rhizoecidae are mealybugs that mostly live underground and feed on plant rootlets and are commonly known as "root mealybugs" or "ground mealybugs" (Williams 1998). The family includes 238 extant species belonging to 19 genera (García Morales et al 2016, Tanaka 2016) of which 69 species have been recorded from the Neotropical Region (Williams \& Granara de Willink 1992, Kozár \& Konczné Benedicty 2007, García Morales et al 2016, Ramos-Portilla \& Caballero 2016). The associated host plants of the rhizoecid mealybug species are often not clearly understood, and when they are known from different host plants, it is unclear whether they have any preference for any plants. According to the collecting method, specimens are often found loosely in the soil, not feeding on any host, and sometimes the same species may be found on the roots of many species of plants (A.A. RamosPortilla, personal observation). When sampling for rhizoecid mealybugs, it is often necessary to pull out the entire root system of the plant in order to determine whether the root on which the specimen was found belongs to the true host. As for species of the genus Rhizoecus, some degree of specialization can be found, where several species prefer xerophilous habitats (Kozár \& Konczné Benedicty 2007).

Within the family Rhizoecidae, the subfamilies Xenococcinae and Rhizoecinae can be easily separated by the absence of ostioles, disc pores, and tubular ducts in the Xenococcinae, which are present in the Rhizoecinae. In the Rhizoecinae, three main lineages (tribes) can be recognized, namely the Geococcini, the Rhizoecini, and the Ripersiellini (Kozár \& Konczné Benedicty 2007). The Geococcini is characterized by having (i) sclerotized anal lobes, (ii) strong setae on the head and posterior abdominal segments, and (iii) modified tritubular ducts. The Rhizoecini has tritubular ducts but without sclerotized anal lobes. Several new evolutionary lines 
are known in this tribe such as Marottarhizoecus Kozár \& Konczné Benedicty which has tritubular ducts surrounded by multilocular pores, Benedictycoccina Kozár \& Foldi which has tritubular ducts surrounded by trilocular pores, and Coccidella Hambleton which has a group of trilocular pore clusters in the middle of the ventral surface.

The Rhizoecini tribe is composed of 112 species grouped into six genera, namely Benedictycoccina Kozár and Foldi (4 spp.), Coccidella (10 spp.), Kissrhizoecus Kozár and Konczné Benedicty (1 sp.), Rhizoecus Künckel d'Herculais (93 spp.), Marottarhizoecus Konczné Benedicty (2 spp.), and Williamsrhizoecus Kozár \& Konczné Benedicty (2 spp.) (Kozár \& Konczné Benedicty 2007, García Morales et al 2016, Kaydan et al 2018).

The genus Rhizoecus has been studied in detail by different authors, some of which treated the genus in a broader sense (Ben-Dov 1994, Williams 1998, 2004, Jansen 2001, 2003) and others who considered the genus in a much narrower sense (Williams 1973, Matile-Ferrero 1976, Tang 1992, Kozár \& Konczné Benedicty 2002, 2003, 2005, 2007, Kozár \& Foldi 2004). According to Kozár and Konczné Benedicty (2007), Rhizoecus is characterized by the combination of the following features: (i) 5 or 6 segmented antennae, (ii) legs well developed, (iii) dorsum and venter with tritubular ducts, and (iv) anal ring with 6 setae.

Rhizoecus has 93 species of which 40 species occur in the Neotropical Region (Kozár \& Konczné Benedicty 2007). In the present paper, two new Rhizoecus species are described from the Neotropical Region. An identification key and new additional locality records for the currently known Rhizoecus species present in the Neotropical Region are provided and discussed.

\section{Material and Methods}

The specimens described and recorded in this study were all obtained from soil samples deposited in the Hungarian Natural History Museum (HNHM) collection (over 5000 samples in total). The samples were extracted by Berlese funnel, an apparatus widely used to extract living organisms, particularly arthropods, which works by creating a temperature gradient over the sample, such that mobile organisms will move away from the higher temperatures and fall into a collecting vessel, where they are preserved for examination (Southwood \& Henderson 2000).

Specimens were prepared for light microscopy using the slide-mounting method discussed by Kosztarab \& Kozár (1988). The morphological terminology used follows Williams (2004), Kozár and Konczné Benedicty (2007), and Ramos-Portilla (2014).
All measurements and counts were taken from all the available material and the values are given as a range for each character.

Type material is deposited in the Plant Protection Institute, Centre for Agricultural Research, Hungarian Academy of Sciences (PPI).

Distribution data for each species have been provided, with new country records signed with an asterisk. For the host plant list of each species, see García Morales et al (2016).

\section{Result and Discussion}

\section{Rhizoecus Künckel d'Herculais}

Type species: Rhizoecus falcifer Künckel d'Herculais, 1878, by monotypy

Neorhizoecus Hambleton, 1946

Radicoccus Hambleton, 1946

Body of adult female elongate to round, usually membranous. Anal lobe poorly or moderately developed, often bearing 3 long apical setae (1 ventral, 2 dorsal), or with numerous short setae. Antennae often placed close together, short, strongly geniculate, each 5 or 6 segmented, segments 2-5 often wider than long, last segment usually longer than wide; in species with 6-segmented antennae, the fifth segment has 1 falcate sensory seta and 4 falcate setae on the sixth antennal segment. In species with 5-segmented antennae, there are usually 5 falcate setae on the terminal segment and 1 falcate seta at the apex of the preapical segment. Labium longer than wide. Legs well developed, tarsus usually shorter than tibia, often with spine-like setae on inner margins, tarsus tapering, with a pair of knobbed digitules at apex. Claw slender and elongate, with short setose or knobbed digitules. Eyes present or absent. Circuli if present numbering 1 to 6 , truncate, conical, elongate, cylindrical, or bulbous, with distal end sometimes flat, reticulated or with minute papilla-like projections. Anterior and posterior ostioles present, sometimes anterior ones absent.

Frons often with a ventral sclerotized cephalic plate, sometimes with a few setae on margin. Body setae usually short and hair-like, often extensively covering surface. Trilocular pores present. Oral collar tubular ducts and multilocular pores with 7-12 loculi present or absent. Anal ring well developed with or without long elongate triangular pores, bearing 6 hair-like setae. Internal female genital organ shows great variability in shape and size (Kozár \& Konczné Benedicty 2007). 
Key to Rhizoecus species distributed in the Neotropics, based on adult females

1 - Multilocular pores present...................................2 - Multilocular pores absent.......................................28

2 - Claw digitules spine-like, shorter than claw..................3 - Claw digitules with blunt apices, as long as claw........................................ amorphophalli (Betrem)

3 - Multilocular disc pores on dorsum present.............4 - Multilocular pores on dorsum absent....................20

4 - Oral collar tubular ducts on dorsum present............5 - Oral collar tubular ducts on dorsum absent...........10

5 - Oral collar tubular ducts in rows on all abdominal segments.

- Oral collar tubular ducts scarce on margin of dorsum only. R. falcifer (Kunckel d'Herculais)

6 - Antennae 6 segmented. ....7

- Antennae 5 segmented.

7 - Multilocular disc pores present on the head and thorax on dorsum. R. iquitosi Konczné Benedicty \& Kozár - Multilocular pores absent on the head and thorax on dorsum. .R. latus (Hambleton)

8 - With a circulus.

R. microtubularis Konczné Benedicty \& Kozár

- Without circulus. ...9

9 - Oral collar tubular ducts present on the head and thorax on venter........R. boliviensis Konczné Benedicty - Oral collar tubular ducts absent on the head and thorax on venter..... R. nitidalis Hambleton

10 - Anal ring outer pores with spiculae... ...11

- Anal ring outer pores without spiculae. .17

11 - Multilocular pores only on abdominal segments on dorsum. ...12 - Multilocular pores on all over the surface on dorsum. ...13

12 - Tritubular ducts short (about two times longer than wide)...... R. stangei McKenzie - Tritubular ducts long (about three-four times longer than wide)...... R. coffeae Laing

13 - Anterior pair of ostioles absent. R. spinipes (Hambleton)

- Anterior pair of ostioles present.

14-One circulus present.

................ R. costaricensis Konczné Benedicty \& Kozár

- Circulus absent.

15 - Ostioles membranous, anal ring outer row pores with more than one spiculae..... ....16 - Ostioles sclerotized, anal ring outer row pores with one spiculus.....................R. americanus (Hambleton)

16 - Tritubular ducts 46-54 altogether on both sides..................................... distinctus (Hambleton) - Tritubular ducts 60-74 altogether on both sides................................... associatus (Hambleton)
17 - Both pair of ostioles present. .18

- Anterior pair of ostioles absent

$R$. granaradewillinkae sp. $\mathrm{n}$.

18 - Ostioles heavily sclerotized .R. caladii Green

- Ostioles membranous. ...19

19 - Multilocular disc pores mostly around vulva, very few on other parts of the venter and dorsum.............................. neomexicanus Mckenzie - Multilocular disc pores in transverse rows and bands on both body surfaces. R. kontschani sp. $\mathrm{n}$.

20-Antennae 5 segmented. .21

- Antennae 6 segmented..................................................24

21 - Oral collar tubular ducts present on dorsum.........22

- Oral collar tubular ducts absent on dorsum...............23

22- Circulus present.

...R. erikae Konczné Benedicty \& Kozár

- Circulus absent........................... R. pauciporus Hambleton

23- Trilocular pores present on dorsum.

R. compotor Williams \& Granara de Willink

- Trilocular pores absent on dorsum.

R. setosus (Hambleton)

24- Multilocular pores 4-10 around vulva...................25

- Multilocular pores 50-60 on at least last 3 abdom-

inal segments.

.26

25-Tritubular ducts short (about two times longer than

wide). R. mayanus (Hambleton)

- Tritubular ducts long (about three-four times longer than wide). $R$. neostangei Miller \& McKenzie

26- Oral collar tubular ducts present on last abdominal segment on venter, scarce... .27

- Oral collar tubular ducts completely absent on venter...... ..R. cyperalis (Hambleton)

27- Tritubular ducts present on venter.

R. nemoralis (Hambleton)

- Tritubular ducts absent on venter. R. subcyperalis Hambleton

28- Tubular ducts present.

- Tubular ducts absent. R. olmuensis Hambleton

29- With two or more circulus. ...30

- With one circulus. .32

30- Three circuli present. R. ovatus Hambleton

- Two circuli present. ..31

31 - Tritubular ducts present on middle of thorax and head on dorsum, oral collar tubular ducts in transverse rows on abdominal segments..............R. polyporus Hambleton - Tritubular ducts absent on middle of thorax and head on dorsum, oral collar tubular ducts very few on abdominal segments.

R. demerarae Williams \& Granara de Willink

$32-$ Tritubular ducts short (about two times longer than wide). ...33 - Tritubular ducts long (about three-four times longer than wide). . .38 
33 - Anal ring pores with spiculae...............................34

- Anal ring without spiculae..............................................35

34-Claw digitules spine-like, shorter than claw...................................... arabicus Hambleton

- Claw digitules with blunt apices, ca. same size as claw........................................ keysensis Hambleton

35 - Anal ring pores in outer row more than 30......................................... R. macgregori Hambleton

- Anal ring pores in outer row less than 26. ..37

36 - Tritubular ducts present on middle part of last dorsal segments. .R. cacticans (Hambleton)

- Tritubular ducts absent on middle part of last dorsal segments. R. leucosomus (Cockerell)

37 - Anal ring pores with spiculae. ...38

- Anal ring pores without spiculae.

38 - Number of tritubular ducts on dorsum between 30 and 40. R. favacirculus Hambleton

- Number of tritubular ducts on dorsum more than 41. R. simplex (Hambleton)

39- Eyes present R. tropicalis Hambleton

- Eyes absent

40-Claw digitules setose, longer than claw. . .41

- Claw digitules setose, shorter than claw........ R. variabilis Hambleton

41 - Tritubular ducts on mid-dorsum present (more than 10)... R. atlanticus (Hambleton)

- Tritubular ducts on middorsum absent or very few (9 or fewer)..... . .42

42 - Anal ring pores in outer row between 20 and

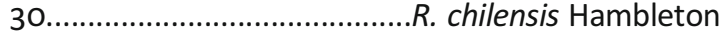
- Anal ring pores in outer row less than 20. R. apizacos Hambleton

\section{Rhizoecus arabicus Hambleton}

Material examined. Argentina: 4 females-Jujuy Province, Ledesma Department, Calilegua National Park, South of Abra de Cañas, moss forest, litter, $23^{\circ} 41.3^{\prime} \mathrm{S}, 64^{\circ} 54.1^{\prime} \mathrm{W}$, 2253 m a.s.I., 05.11.2006, leg. Sziráki Gy, Horváth E, González Olazo E (HNHM D-Am 481; PPI: 12433)

Distribution. Bolivia, Colombia, Costa Rica, Guadeloupe, Mexico, Peru, Trinidad and Tobago, USA (García Morales et al 2016); *Argentina

\section{Rhizoecus boliviensis Konczné Benedicty}

Material examined. Bolivia: 2 females-Beni Department, Guayaramerin, Estancia Esperanza, banana plantation, dry, decaying roots below preceding layer, 07.12.1965, leg. Balogh J, Mahunka S, Zicsi A (HNHM D-Am 2866; PPI: 12790); 1 female-same place and collectors, cacao plantation, lower, rooty horizon of leaf litter, 10.10.1965 (HNHM D-Am 2868; PPI: 12461); 1 female-same place and collectors, untouched forest, humid leaf litter, 10.10.1965 (HNHM D-Am 2869; PPI: 12462); 3 females-same place and collectors, gallery forests along the Mamore river, lower horizon of leaf litter, 10.10.1965 (HNHM D-Am 2872; PPI: 12463)

Distribution. Bolivia (Kozár \& Konczné Benedicty 2007)

\section{Rhizoecus cacticans (Hambleton)}

Material examined. Bolivia: 1 female-Beni Department, Guayaramerin, Estancia Esperanza, untouched forest, humid leaf litter, 10.10.1965, leg. Andrásy I, Balogh J, Loksa I, Mahunka S, Zicsi A (HNHM D-Am 2869; PPI: 12462). Chile: 1 female-Provincia Santiago, Tiltil, Cuesta la Dormida, drier parts of valley side, soil, 05.11.1965, leg. Balogh J, Mahunka S, Zicsi A (HNHM D-Am 2861; PPI: 12791)

Distribution. Argentina, Australia, Chile, Colombia, Czech Republic, Denmark, Ecuador, France, Germany, Greece, Guatemala, Honduras, Hungary, Italy (mainland and Sicily), Netherlands, Norway, Peru, Poland, Russia, Spain (Canary Islands), UK, USA (García Morales et al 2016); *Bolivia

\section{Rhizoecus costaricensis Konczné Benedicty \& Kozár}

Material examined. Brazil: 1 female-Maranhão State, northwest from Imperatriz, Serra do Gurupi, Fazenda Agua Azul, leaf litter, 09.09.1967, leg. Balogh J (HNHM D-Am 2916; PPI: 12793). Colombia: 2 females-Rio Carlo, leaf litter and moss, 02.10.1984, leg. Zicsi A, Loksa I (HNHM Colombia, 1984.X.; PPI: 12431). Costa Rica: 3 females-Heredia Province, La Selva Biological Station, River Sura, primary rain forest, $800 \mathrm{~m}$ a.s.l., 14.01.1992, leg. Balogh J (HNHM CR92.B21; PPI: 12792). Venezuela: 1 female-Maracay University, park, leaf litter and root matrix, 30. 08.1973 (HNHM D-Am 3311; PPI: 12794)

Distribution. Bolivia, Costa Rica (Kozár \& Konczné Benedicty 2007), *Colombia, *Venezuela

Rhizoecus demerarae Williams and Granara de Willink

Material examined. Argentina: 3 females-Jujuy Province, Ledesma Department, Calilegua National Park, South of Abra de Cañas, moss forest, litter and moss, $1700 \mathrm{~m}$ a.s.l., 05.11.2006, leg. Sziráki Gy, Horváth E, González Olazo E (HNHM D-Am 469; PPI: 12798); 1 female-same data (HNHM D-Am 470. PPI: 12799). Brazil: 2 females-São Paulo State, Campinas, Americana, leaf litter, 21.09.1967, leg. Balogh J (HNHM D-Am 2927; PPI: 12803), 1 female-Rio de Janeiro State, Itatiaia National Park, Itaporani rainfall, primary rain forest, leaf litter, soil and moss, 27.05.1992, leg. Balogh J (HNHM BR92.B.5; PPI: 12795); 2 females-Mato Grosso State, Pantanal, Fazenda Uberaba, Garon Maya, leaf litter, leg. Balogh J (HNHM BR92.B.55; PPI: 12797); 1 female- 
São Paolo State, São Roqūe, Project Itatūba, Eucalyptus plantation, leaf litter, $850 \mathrm{~m}$ a.s.l., 09.01.1995, leg. Balogh J (HNHM BR95.B17; PPI: 12796); 5 females-São Paolo State, São Roque, Project Itatuba, Sapucaia Lake, submontane rain forest, leaf litter, $800 \mathrm{~m}$ a.s.l., 09.01.1995, leg. Balogh J (HNHM BR95.B21; PPI: 12396). Colombia: 1 female-Rio Carlo, leaf litter and moss, 02.10.1984, leg. Zicsi A, Loksa I (HNHM Colombia, 1984; PPI: 12432). Ecuador: 1 femalePichincha Province, above Quito, $46 \mathrm{~km}$ leaving Quito to Santo Domingo, soil from 15-cm depth, 3200-3400 m a.s.l., 21.04.1988, leg. Zicsi A, Csuzdi Cs (HNHM D-Am 634; PPI: 12800); 2 females-Pichincha Province, on the way from Tandajapa to Nono, rainforest, moss, 08.04.1987, leg. Zicsi A, Loksa I (HNHM D-Am 715; PPI: 12802); 1 female-Azuay Province, leaving Chordeleg, $39 \mathrm{~km}$ from Cuenca, moss, 03.05.1988, leg. Zicsi A, Csuzdi Cs (HNHM D-Am 676; PPI: 12801); 4 females-Azuay Province, $2 \mathrm{~km}$ leaving Sigsig, moss from the slope of bank, 03.05.1988, leg. Zicsi A, Csuzdi Cs (HNHM D-Am 687; PPI: 12448)

Distribution. Brazil, Guayana, Peru (García Morales et al 2016); *Argentina, *Colombia, *Ecuador

\section{Rhizoecus distinctus (Hambleton)}

Material examined. Costa Rica: 2 females-Heredia Province, La Selva Biological Station, River Sura, primary rain forest, 800 m a.s.l., 14.01.1992, leg. Balogh J (HNHM Cr92.B.21; PPI: 12804); 1 female-Talamanca Mt. Range, Sierra de La Muerte, El Empalme, lower mountain wet forest, leaf litter and root matrix, $2150 \mathrm{~m}$ a.s.l., 24.01.1992, leg. Balogh J (HNHM Crg2.B.61; PPI: 12805)

Distribution. USA (García Morales et al 2016); *Costa Rica

\section{Rhizoecus falcifer Künckel d' Herculais}

Material examined. Chile: 1 female-Tarapaca Province, Misituni, Berlese-samples from 12 points in cross-section of smaller valley running at right angles to Rio Lauca, 25.11.1965, leg. Andrásy I, Balogh J, Loksa I, Mahunka S, Zicsi A (HNHM DAm 2716; PPI: 12809). Costa Rica: 2 females-Alajuela Province, Arenal volcano, hot springs, secondary rain forest leaf litter, $2800 \mathrm{~m}$ a.s.l., 16.01.1993, leg. Balogh J (HNHM CR93.B112; PPI: 12806); 1 female-Puntarenas Province, Manuel Antonio National Park, secondary rain forest, leaf litter and root matrix, 24.01.1993, leg. Balogh J (HNHM CR93.B133; PPI: 12807); 1 female-same data (HNHM CR93 B136; PPI: 12419); 5 females-same data (HNHM CR93 B141; PPI: 12421). Ecuador: 1 female-Prov. Azuay, $26 \mathrm{~km}$ from Cuenca, grass, 26.04.1988, leg. Zicsi A, Csuzdi Cs (HNHM DAm 599; PPI: 12808)

Distribution. Algeria, Australia, Czech Republic, France, Hungary, Ireland, Italy (mainland and Sicily), Malta, Mexico, Morocco, New Zealand, Saint Helena, South Africa, Spain (mainland and Canary Islands), Suriname, Trinidad and Tobago, Trinidad, UK, England, USA (García Morales et al 2016); *Chile, *Costa Rica, *Ecuador

Rhizoecus granaradewillinkae Kaydan \& Szita sp. n. (Fig 1)

Material examined. Costa Rica: Holotype: 1 female-Alajuela Province, Arenal volcano, Northern slopes, rainforest, moss from trees, 400-500 m a.s.l., 16.01.1993, leg. Balogh J (HNHM CR93.B108; PPI: 12837)

Distribution. Costa Rica

Description. Slide-mounted adult female

Adult female. Body elongate-oval, $0.6 \mathrm{~mm}$ long, $0.3 \mathrm{~mm}$ wide. Eyes marginal, 8-10 $\mu \mathrm{m}$ wide. Antenna 5 segmented, 115-120 $\mu \mathrm{m}$ long, with 4 fleshy setae, on apical segment each seta 25-28 $\mu \mathrm{m}$ long; apical segment 40-43 $\mu \mathrm{m}$ long, $22.5 \mu \mathrm{m}$ wide, with apical setae each $25-28 \mu \mathrm{m}$ long. Clypeolabral shield $75 \mu \mathrm{m}$ long, $70 \mu \mathrm{m}$ wide. Labium 3 segmented, $50 \mu \mathrm{m}$ long, $45 \mu \mathrm{m}$ wide. Anterior spiracles each 15-18 $\mu \mathrm{m}$ long, 7-10 $\mu \mathrm{m}$ wide across atrium; posterior spiracles each 20-25 $\mu \mathrm{m}$ long, 7-10 $\mu \mathrm{m}$ wide across atrium. Legs well developed, length data for posterior legs: coxa $45-60 \mu \mathrm{m}$, trochanter + femur $90 \mu \mathrm{m}$, tibia + tarsus 102-105 $\mu \mathrm{m}$, claw $15-18 \mu \mathrm{m}$. Ratio of lengths of tibia + tarsus to trochanter + femur 1.13-1.16:1; ratio of lengths of tibia to tarsus 1.00-1.40:1; ratio of length of hind trochanter + femur to greatest width of femur 2.00-2.04:1. Claw digitules spine-like, $5 \mu \mathrm{m}$ long. Anterior ostioles not detected; posterior ostioles without trilocular pores and with only 3 setae. Anal ring $40.0 \mu \mathrm{m}$ wide, bearing 6 setae, each seta 75-95 $\mu \mathrm{m}$ long

Dorsum. Derm membranous, without any cerarii around body margin. Setae on anal lobe hair-like, each 40-45 $\mu \mathrm{m}$ long; body setae short, flagellate, each 10-25 $\mu \mathrm{m}$ long, scattered on the head, thorax, and abdominal segments. Trilocular pores each 2.0-2.5 $\mu \mathrm{m}$ in diameter, scattered over the entire body. Multilocular disc pores on abdominal segments as follows: segments I-III, 27; IV, 9; V, 8; VI, 8; VII, 9; $\mathrm{VIII}+\mathrm{IX}, 0$; and about 74 scattered on the head and thorax; each pore 9-10 $\mu \mathrm{m}$ in diameter. Tritubular ducts, each duct 7-8 $\mu \mathrm{m}$ wide at mid-width, on abdominal segments as follows: segment I: 5, II: 6, III: 5, IV: 5, V: 6, VI: 5, VII: 5, VIII + IX: 2; and 30 on the thorax and head; each pore 8-9 $\mu \mathrm{m}$ in diameter

Venter. Setae flagellate, each $10-25 \mu \mathrm{m}$ long, longest setae situated medially on the head. Apical setae of anal lobe each 55-60 $\mu \mathrm{m}$ long. Multilocular disc pores on abdominal segments as follows: segments I-III, 30; IV, 9; V, 9; VI, 7; VII, 12; VIII + IX, 6; and scattered on the head and thorax: 84; each pore $7-8 \mu \mathrm{m}$ in diameter. Trilocular pores, each $2.5 \mu \mathrm{m}$ in diameter, scattered on venter. Tritubular ducts, each duct $5 \mu \mathrm{m}$ wide at mid-width, present in a single row across 


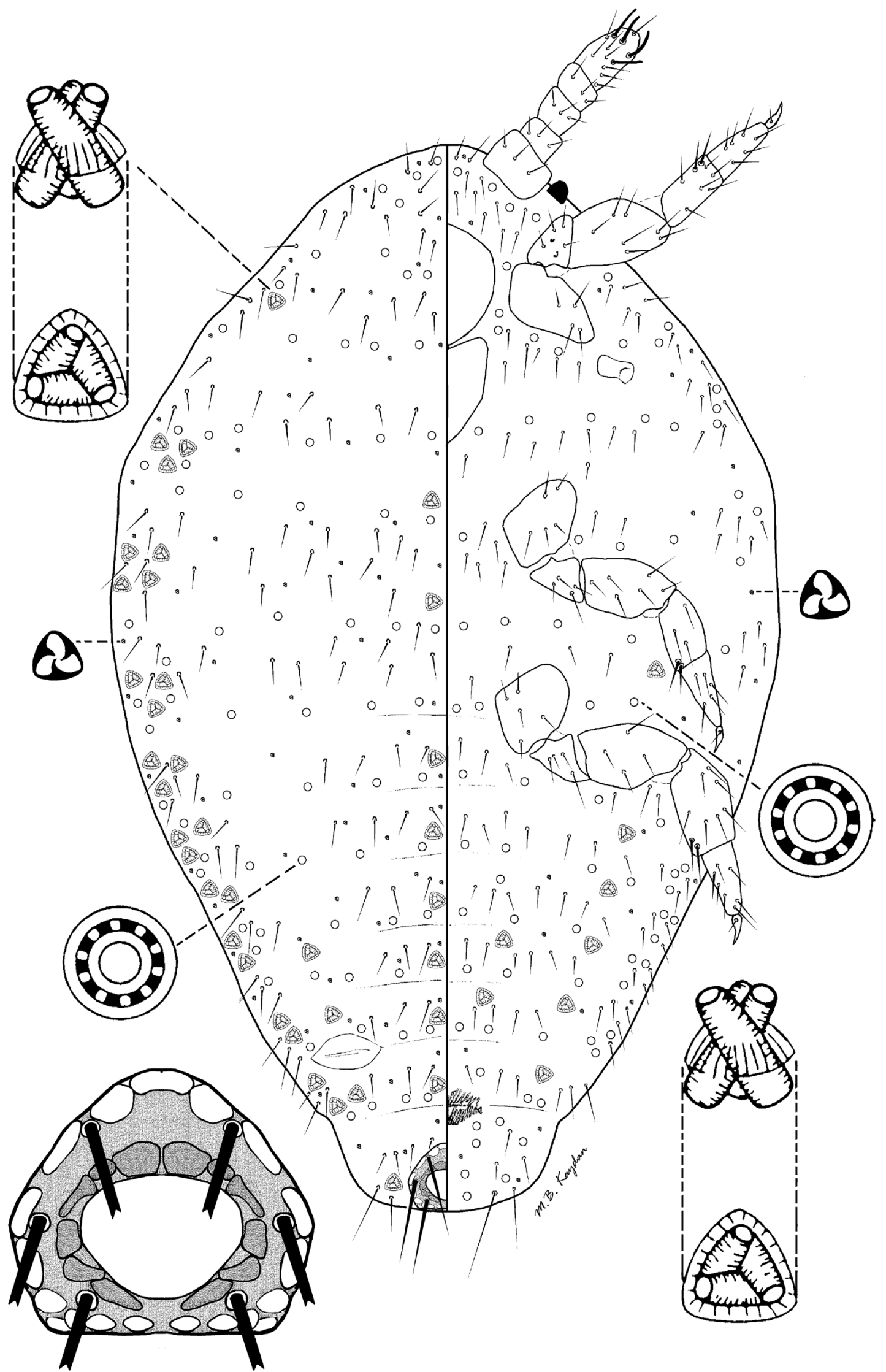

Fig 1 Rhizoecus granaradewillinkae Kaydan and Szita sp. n., adult female. 
abdominal segments, as follows: segments I-III, 2; IV, 6; V, 5; $\mathrm{VI}, 5 ; \mathrm{VII}, 5 ; \mathrm{VIII}+\mathrm{IX}, 2$

Etymology. The species was named in honor of the Argentinean coccidologist, María Cristina Granara de Willink, who made significant contributions to the knowledge of the taxonomy and fauna of South American scale insect species.

Comments. Rhizoecus granaradewillinkae Kaydan \& Szita is characterized by having (i) five segmented antennae, (ii) claw digitules spine-like, (iii) only posterior pairs of ostioles present, (iv) multilocular pores present on both venter and dorsum, and ( $v$ ) absence of oral collar tubular ducts on both sides. Rhizoecus granaradewillinkae is most similar to $R$. distinctus (Hambleton) and $R$. associatus (Hambleton) in having multilocular pores on dorsum and spine-like claw digitules shorter than claw, while the new species Rhizoecus granaradewillinkae differs from all the above species in lacking anterior ostioles.

\section{Rhizoecus keysensis Hambleton (Fig 2)}

Material examined. Chile: 2 females-Provincia Valparaiso, Concón, $5 \mathrm{~km}$ from Concón on the road leading to Quintero, sand dunes, beneath, tean-tree, 10.10.1965, leg. Andrásy I, Balogh J, Loksa I, Mahunka S, Zicsi A (HNHM DAm 2726; PPI: 12457). Ecuador: 2 females-Pichincha Province, Pululagua crater and its surroundings, Mitad del Mundo, moss from under bushes growing on the sides of the hollow, 12.02.1986, leg. Zicsi A, Loksa I (HNHM D-Am 592; PPI: 12434); 1 female-Azuay Province, between Giron and Victoria del Portete, leaf litter, 02.05.1988, leg. Zicsi A, Csuzdi Cs (HNHM D-Am 686; PPI: 12810)

Distribution. USA (García Morales et al 2016), *Chile, *Ecuador

Rhizoecus kontschani Kaydan \& Konczné Benedicty sp. n. (Fig 3)

Material examined. Holotype: Brazil-1 female-Rio de Janeiro State, Itatiaia National Park, Itaporani rainfall, moss, 27.05.1992, leg. Balogh J (HNHM BR92.B9; PPI: 12360). Paratypes: 4 females in 3 slides $(1,1,2)$-same data as holotype; 8 females in 3 slides $(3,3,2)$-São Paolo State, Ilha do São Sebastiaõ, protected urban forest, leaf litter, 29.05.1992, leg. Balogh J (HNHM BR92.B14; PPI: 12366); 1 female-São Paolo State, Caraguatatuba, Serra do Mar State Park, Atlantic rain forest, moss, 900-1000 m a.s.l., 03.06.1992, leg. Balogh J (HNHM Br92.B39; PPI: 12831)

Distribution. Brazil

Description. Slide-mounted adult female

Adult female. Body elongate-oval, 0.80-1.56 mm long, $0.42-0.94 \mathrm{~mm}$ wide. Eyes marginal, 7.5-10.0 $\mu \mathrm{m}$ wide.
Antenna 6 segmented, 150-170 $\mu \mathrm{m}$ long; apical segment 35-45 $\mu \mathrm{m}$ long, 30-35 $\mu \mathrm{m}$ wide, with 4 fleshy setae (plus 1 fleshy seta on fifth segment), each seta $30-40 \mu \mathrm{m}$ long and apical setae each 25.0-27.5 $\mu \mathrm{m}$ long. Clypeolabral shield $85-$ $100 \mu \mathrm{m}$ long, 85.0-87.5 $\mu \mathrm{m}$ wide. Labium 3 segmented, 95$100 \mu \mathrm{m}$ long, 60-65 $\mu \mathrm{m}$ wide. Anterior spiracles each 32.0$35 \mu \mathrm{m}$ long, 12-15 $\mu \mathrm{m}$ wide across atrium; posterior spiracles each $35-38 \mu \mathrm{m}$ long, $15-20 \mu \mathrm{m}$ wide across atrium. Legs well developed, length data for posterior legs: coxa 85-100 $\mu \mathrm{m}$, trochanter + femur 140-160 $\mu \mathrm{m}$, tibia + tarsus 130-165 $\mu \mathrm{m}$, claw $30-35 \mu \mathrm{m}$. Ratio of lengths of tibia + tarsus to trochanter + femur 1.01-1.00:1; ratio of lengths of tibia to tarsus 0.96-1.0:1; ratio of length of hind trochanter + femur to greatest width of femur 2.30-3.1:1. Claw digitules spine-like, $3 \mu \mathrm{m}$ long. Both pairs of ostioles present; anterior ostioles each with a total for both lips of 10-16 trilocular pores and 610 setae; posterior ostioles each with a total for both lips of 12-18 trilocular pores and 7-10 setae. Anal ring 45-55 $\mu \mathrm{m}$ wide, bearing 6 setae, each seta 65-90 $\mu \mathrm{m}$ long

Dorsum. Derm membranous, without any cerarii around body margin. Setae on anal lobe hair-like, each 55-60 $\mu \mathrm{m}$ long; body setae short, flagellate, each 10-25 $\mu \mathrm{m}$ long, scattered on the head, thorax, and abdominal segments. Trilocular pores each 2-3 $\mu \mathrm{m}$ in diameter, scattered over the entire body. Multilocular disc pores on abdominal segments numbering as follows: segment I, 8-11; II, 10 or 11; III, 9-13; IV, 7-9; V, 5-9; VI, o-2; VII, o-2; VIII + IX, o-4; 72-97 pores scattered on the thorax and head, each pore $6-8 \mu \mathrm{m}$ in diameter. Tritubular ducts present over the entire body and form a submarginal and a median row, having 3 or 4 ducts on each abdominal segments, except on segment VIII + IX, each duct 10-12 $\mu \mathrm{m}$ wide on medial row

Venter. Setae flagellate, each 10-35 $\mu \mathrm{m}$ long, longest setae situated medially on the head. Apical setae of anal lobe each 55-60 $\mu \mathrm{m}$ long. Multilocular disc pores on abdominal segments numbering as follows: segments I-III, 42-44; IV, 13-15; V, 18-28; VI, 23-30; VII, 41-48; VIII + IX, 44-46; 81-102 pores scattered on the thorax and head, each pore $7-8 \mu \mathrm{m}$ in diameter. Trilocular pores, scattered, each $2.0-2.5 \mu \mathrm{m}$ in diameter. Tritubular ducts, each 7.5-8.0 $\mu \mathrm{m}$ wide at midwidth, present in a single row across abdominal segments, as follows: segment IV, $5 ; \mathrm{V}, 7 ; \mathrm{VI}, 8$; on margin of segment VII, 4-6; VIII + IX, 2; I-III, 10-12; and head and thorax 10-14

Etymology. The species was named after the Hungarian acarologist, Jenő Kontschán, who gave great motivation to work on South American materials.

Comments. Rhizoecus kontschani Kaydan \& Konczné Benedicty is characterized by having (i) six segmented antennae, (ii) claw digitules spine-like, (iii) anterior and posterior pairs of ostioles present, (iv) multilocular pores present on both venter and dorsum, and (v) absence of oral collar tubular ducts on both sides. Rhizoecus kontschani Kaydan \& Konczné Benedicty is closely related to $R$. spinipes (Hambleton), 

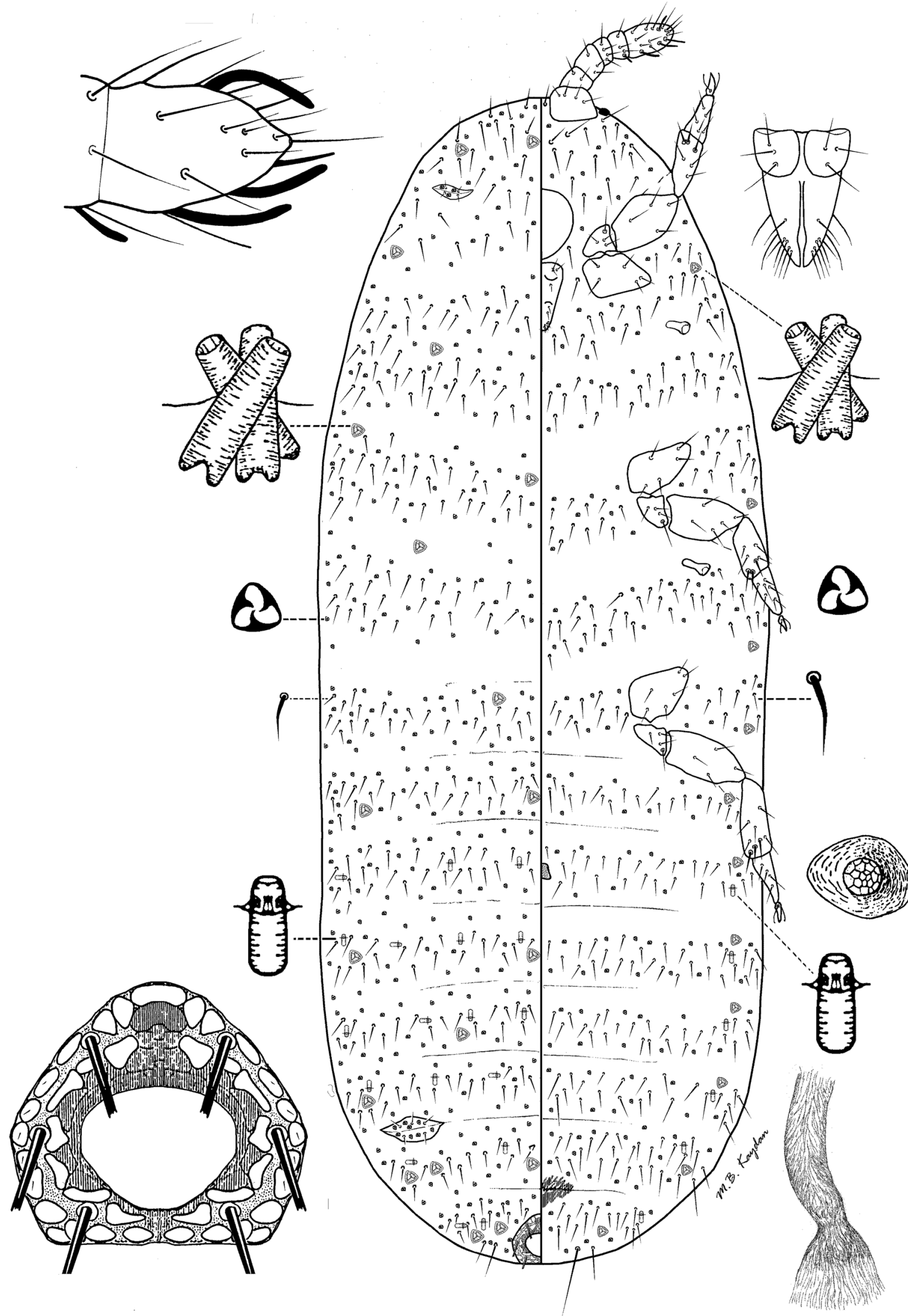

Fig 2 Rhizoecus keysensis Hambleton, adult female. 


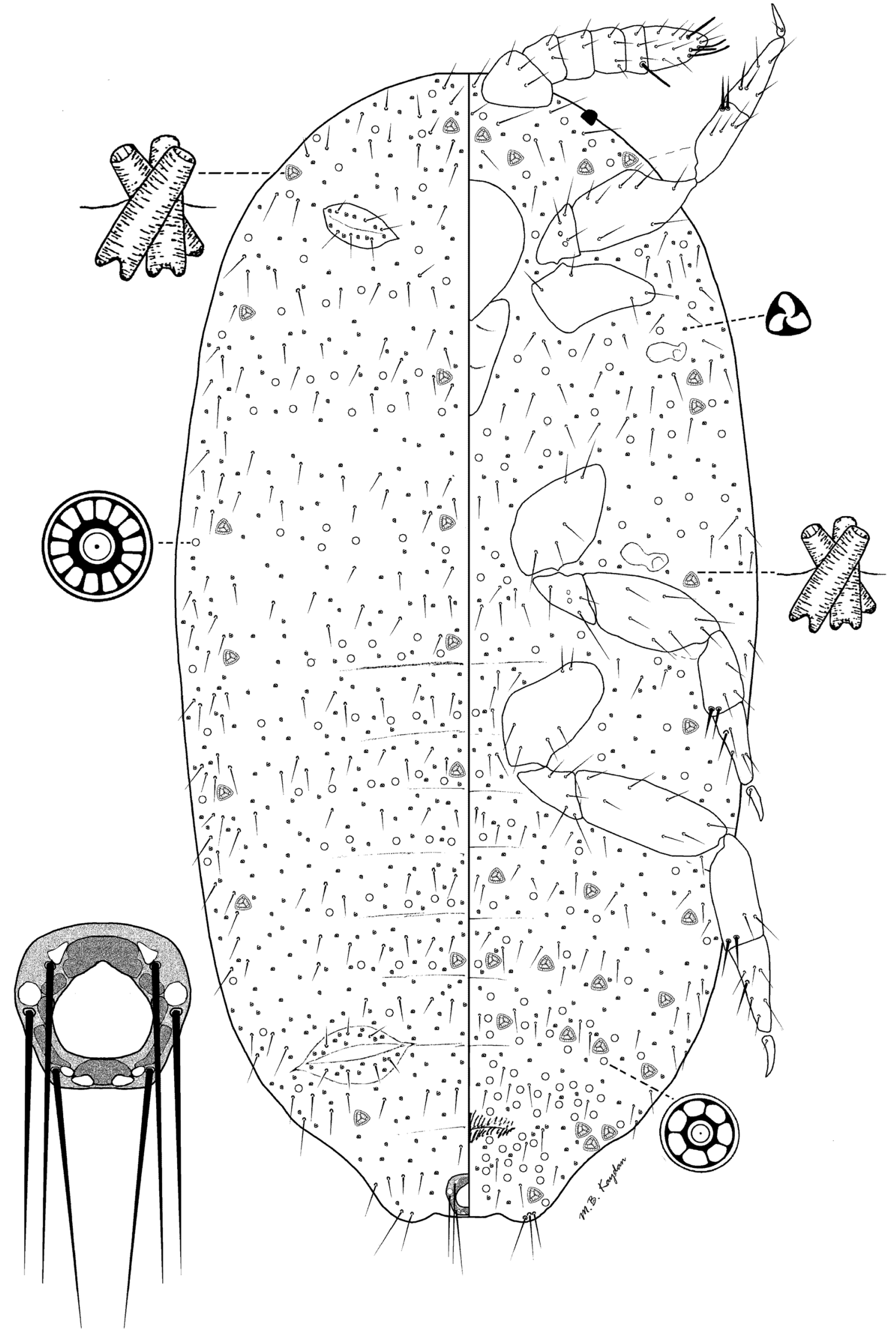

Fig 3 Rhizoecus kontschani Kaydan and Konczné Benedicty sp. n., adult female. 
R. distinctus (Hambleton), and R. associatus (Hambleton), in having multilocular pores on dorsum, spine-like claw digitules shorter than claw and lacking tubular ducts. But the new species Rhizoecus kontschani Kaydan \& Konczné Benedicty differs from all the above species in having high number or multilocular disc pores scattered on dorsum.

\section{Rhizoecus macgregori Hambleton}

Material examined. Bolivia: 2 females - La Paz Department, Caranavi Province, between Corocio and La Paz, after Umduari, shrubby vegetation, leaf litter and moss, 3200 m a.s.l., 16.11.1971, leg. Balogh J (HNHM D-Am 2986; PPI: 12821); 1 female-La Paz Department, Caranavi Province, between Puerto Linares and Caranavi, $41 \mathrm{~km}$ from Puerto Lineares, mountain forest, leaf litter, 14.11.1971, leg. Balogh J (HNHM D-Am 2993; PPI: 12822); 1 female-same data (HMHM D-Am 2994. PPI: 12823); 1 female-same data (HNHM D-Am 2995; PPI: 12824). Chile: 1 female-Santiago Province, Maipu, Quebrada, La Plata, Pundo: La Rinconada, $25 \mathrm{~km} \mathrm{SW}$ from Santiago de Chile, leaf litter from thick, dry forest along brook, 28.09.1965 (HNHM D-Am 2755; PPI: 12819); 2 females-Valparaiso Province, Concón, $5 \mathrm{~km}$ from Concón on the road leading to Quintero, bank of lake among sand dunes, leaf litter of lake-side trees, 10.10.1965, leg. Andrásy I, Balogh J, Loksa I, Mahunka S, Zicsi A (HNHM DAm 2726; PPI: 12458); 1 female-same data (HNHM D-Am 2735; PPI: 12818); 1 female-Coquimho Provincia, Los Villos, Berlese-samples from jungle, leaf litter and soil from drier spot, 05.12.1965, leg. Andrásy I, Balogh J, Loksa I, Mahunka S, Zicsi A (HNHM D-Am 2836; PPI: 12820)

Distribution. USA (Hambleton 1976), *Chile, *Ecuador

\section{Rhizoecus microtubularis Konczné Benedicty \& Kozár}

Material examined. Costa Rica: 1 female-Alajuela Province, Poas Volcano National Park, leaf litter and soil, $1800 \mathrm{~m}$ a.s.l., 21.01.1992., leg. Balogh J (HNHM CR 92.B 51; PPI: 12404); 1 female-Talamanca Mt. Range, Sierra de La Muerte, Alto de la Gloria, lower mountain wet forest, leaf litter, $1800 \mathrm{~m}$ a.s.l., 24.01.1992, leg. Balogh J (HNHM CR 92.B54; PPI: 12811); 1 female-same data, dry leaf litter of epiphytons (HNHM CR 92.B60; PPI: 12812)

Distribution. Costa Rica, Mexico (Kozár \& Konczné Benedicty 2007)

\section{Rhizoecus neostangei Miller \& McKenzie}

Material examined. Brazil: 3 females-Serra do Mar Mt. Range, Rio de Janeiro State, near Paraty, Atlantic forest, leaf litter, $300 \mathrm{~m}$ a.s.l., 05.12.1990, leg. Balogh J (HNHM Br9o.B.95; PPI: 12813)

Distribution. Mexico (García Morales et al 2016), *Brazil

\section{Rhizoecus nitidalis Hambleton}

Material examined. Brazil: 1 female-Serra do Mar Mt. Range, Rio de Janeiro State, near Paraty, Atlantic forest, leaf litter, $300 \mathrm{~m}$ a.s.l., 05.12.1990, leg. Balogh J (HNHM Brgo.B94; PPI: 12814)

Distribution. Brazil (García Morales et al 2016)

\section{Rhizoecus ovatus Hambleton}

Material examined. Bolívia: 1 female-La Paz Department, Caranavi Province, between Corocio and La Paz, moss forest, litter, $2800 \mathrm{~m}$ a.s.l., 16.11.1971, leg. Balogh J (HNHM D-Am 2971; PPI: 12815)

Distribution. Mexico (García Morales et al 2016), *Bolivia

\section{Rhizoecus polyporus Hambleton}

Material examined. Bolivia: 2 females-Beni Dept., $10 \mathrm{~km} \mathrm{~W}$ of Guayaramerin, along the road to Riberalta, virgin forest, sandy soil, leaf litter and root matrix, 28.11.1966, leg. Balogh J (HNHM D-Am 2878; PPI: 12817). Brazil: 1 female-Sao Paolo State, Sao Roqūe, Project Itatūba, Eucalyptus plantation, leaf litter, 09.01.1995, leg. Balogh J (HNHM BR95.B19; PPI: 12816)

Distribution. Mexico (García Morales et al 2016), *Bolivia, *Brazil

\section{Rhizoecus pseudocacticans Hambleton (Fig 4)}

Material examined. Bolivia: 2 females-La Paz Department, Caranavi Province, between Corocio and La Paz, after Umduari, shrubby vegetation, leaf litter and moss, 3200 m a.s.l., 16.11.1971, leg. Balogh J (HNHM D-Am 2986; PPI: 12821); Chile: 3 females-Valparaiso Province, Concón, $5 \mathrm{~km}$ from Concón on the road leading to Quintero, bank of lake among sand dunes, leaf litter of lake-side trees, 10.10.1965, leg. Andrásy I, Balogh J, Loksa I, Mahunka S, Zicsi A (HNHM D-Am 2726; PPI: 12458); 1 female-same data (HNHM D-Am 2735; PPI: 12818); 2 females-Coquimho Provincia, Los Villos, Berlese-samples from jungle, leaf litter and soil from drier spot, 05.12.1965, leg. Andrásy I, Balogh J, Loksa I, Mahunka S, Zicsi A (HNHM D-Am 2836; PPI: 12820)

Distribution. USA (García Morales et al 2016), no. 4298\}, Spain (Canary Islands) (Kaydan et al 2016), *Bolivia, *Chile

\section{Rhizoecus setosus (Hambleton)}

Material examined. Bolivia: 1 female-La Paz Department, Caranavi Province, between Puerto Linares and Caranavi, $41 \mathrm{~km}$ from Puerto Lineares, mountain forest, leaf litter, 14.11.1971, leg. Balogh J (HNHM D-Am 2994; PPI: 12826). Ecuador: 2 females-Napo Province, Rio Jondachi, somewhat after leaving Jondachi, riverside forest, soil and leaf litter, 


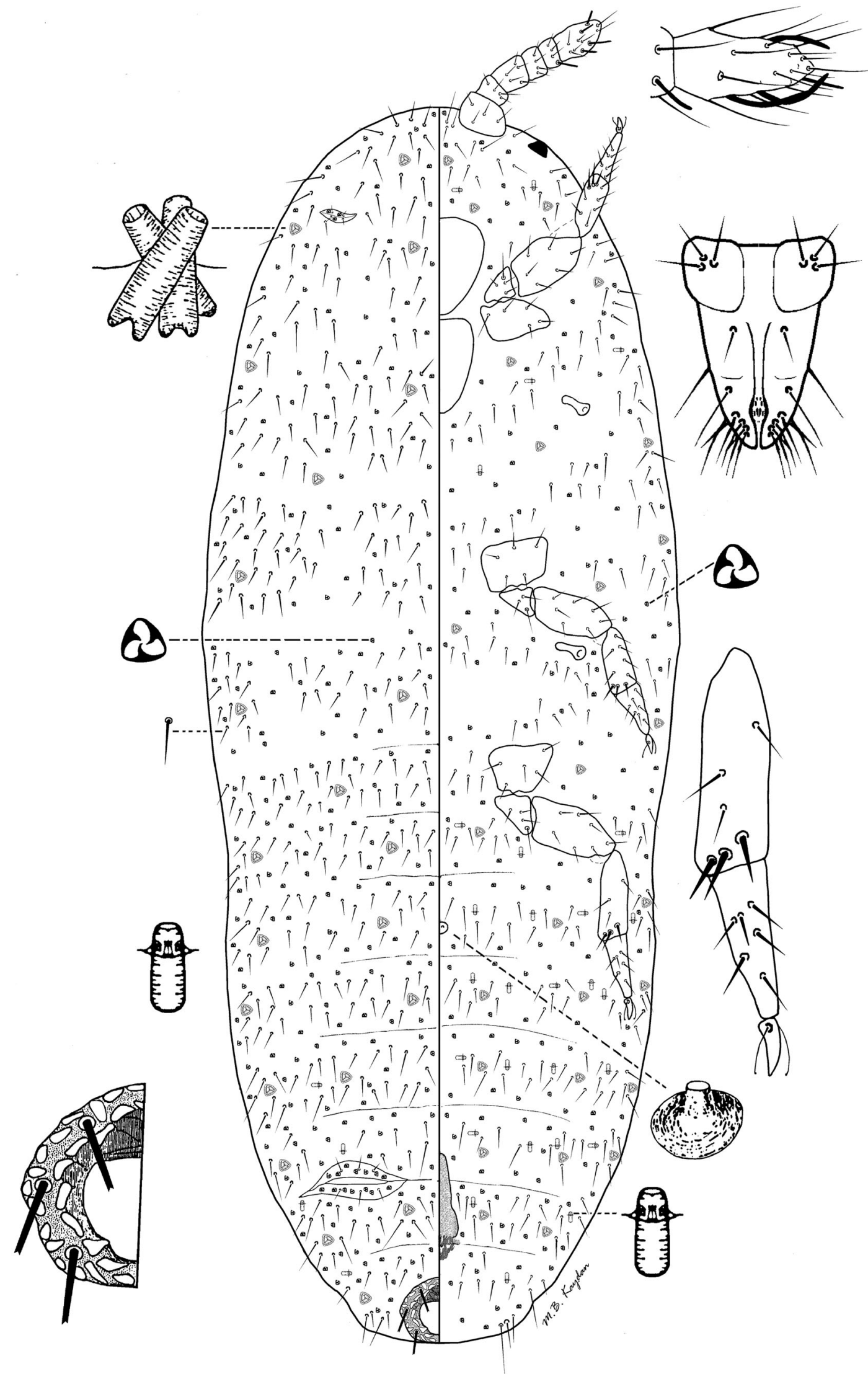

Fig 4 Rhizoecus pseudocacticans Hambleton, adult female. 
10.04.1987., leg. Zicsi A, Loksa I (HNHM D-Am 709PPI: 12825); 2 females-same data (HNHM D-Am 729; PPI: 12456). Peru: 2 females-Lima-Pucallpa transect, $212 \mathrm{~km}$ from Pucallpa, mountain rain forest, moss and leaf litter, 02-04.11.1971, leg. Balogh J (HNHM D-Am 3030; PPI: 12827); 1 femalesame data (HNHM D-Am 3031; PPI: 12828)

Distribution. Colombia, Ecuador, Peru (García Morales et al 2016), *Bolivia

\section{Rhizoecus spinipes Hambleton}

Material examined. Costa Rica: 1 female-Talamanca Mt. Range, Sierra de La Muerte, Alto de la Gloria, dry leaf litter of epiphytons, 1800 m a.s.l., 24.01.1992, leg. Balogh J (HNHM CR92.B60; PPI: 12835); 4 females-Cartago Province, Turrialba, Tropical Agronomy Research and Learning Center (CATIE), moss from trunks, $150 \mathrm{~m}$ a.s.l.,12.01.1993, leg. Balogh J (HNHM CR93.B74. PPI: 12833); 1 female-Limón Province, near Guápiles, Atlantic rain forest, leaf litter and root matrix, 400-500 m a.s.l., 12.01.1993, leg. Balogh J (HNHM CR93.B82; PPI: 12834); 1 female-Puntarenas Province, Manuel Antonio National Park, secondary rain forest, leaf litter and root matrix, 24.01.1993, leg. Balogh J (HNHM CR93.B141; PPI: 12424)

Distribution. Mexico, USA (García Morales et al 2016), *Costa Rica

\section{Rhizoecus subcyperalis Hambleton}

Material examined. Costa Rica: 1 female-San José Province, Cerro La Muerte, mountain rain forest, moss and leaf litter, $3400 \mathrm{~m}$ a.s.l., 24.01.1992, leg. Balogh J (HNHM CR92.B69; PPI: 12838)

Distribution. USA (García Morales et al 2016), *Costa Rica

\section{Rhizoecus variabilis Hambleton}

Material examined. Brazil: 1 female-São Paolo State, Sao Roqūe, Project Itatūba, Eucalyptus plantation, leaf litter, 09.01.1995, leg. Balogh J (HNHM Br95.B19; PPI: 12839); 3 females-Serra del Caldas Navas, Serrado, Caldas Navas, soil and root matrix, 24.01.1995, leg. Balogh J (HNHM Br95.B55; PPI: 12840); 1 female-Rio de Janeiro State, Itatiaia National Park, Itaporani rainfall, rain forest, leaf litter, $1400 \mathrm{~m}$ a.s.l.,16.02.1995, leg. Balogh J (HNHM BR95.B83; PPI code: 12836). Chile: 2 females-Tarapaca Province, Misituni, Berlese-samples from 12 points in cross-section of smaller valley running at right angles to Rio Lauca,,25.11.1965, leg. Andrásy I, Balogh J, Loksa I, Mahunka S, Zicsi A (HNHM DAm 2716; PPI code: 12776); 1 female-Santiago Province, El Arrayan, $10 \mathrm{~km}$ E from Santiago de Chile, fern stems and moss from the ground, 9.10.1965, leg. Andrásy I, Balogh J, Loksa I, Mahunka S, Zicsi A (HNHM D-Am 2724; PPI: 12843); Ecuador: 2 females-Loja Province, leaving Saraguro, $175 \mathrm{~km}$ from Cuenca, moss, 26.04.1988, leg. Zicsi A \& Csuzdi Cs (HNHM D-Am 612; PPI: 12841); 1 female-same data, soil (HNHM D-Am 621; PPI: 12842)

Distribution. Colombia, Guadalupe (García Morales et al 2016), *Brazil, *Chile, *Ecuador

Acknowledgements The authors wish to thank Dr. László Dányi, the curator of the Soil Zoological Collection of the Hungarian Natural History Museum, and Edit Horváth, assistant at the abovementioned museum, for their kind help and for making it possible for us to study the soil sample collection. Special thanks to the anonymous reviewers for their comments on the manuscript. The last author (ÉS) and this project was supported by the János Bolyai Research Scholarship of the Hungarian Academy of Sciences. Open access funding provided by MTA Centre for Agricultural Research (MTA ATK).

Compliance with Ethical Standards All applicable international, national, and institutional guidelines for the collection of biological materials were followed.

Authors Contribution Statement MBK conceived of the study, participated in its design, performed the drawings and measurements, and helped to draft the manuscript. ZKB participated in the design and performed the slide mounting. TK participated in the design of the study and drafted the manuscript as expert of South American scale insects. AARP participated in the design of the study and drafted the manuscript as an expert of Neotropic Rhizoecidae. Final approval of the version to be submitted: Joanne E. Mortimer. ÉS conceived of the study, and participated in its design and coordination and drafted the manuscript. All authors read and approved the final manuscript

Open Access This article is distributed under the terms of the Creative Commons Attribution 4.0 International License (http:// creativecommons.org/licenses/by/4.0/), which permits unrestricted use, distribution, and reproduction in any medium, provided you give appropriate credit to the original author(s) and the source, provide a link to the Creative Commons license, and indicate if changes were made.

\section{References}

Ben-Dov Y (1994) A systematic catalogue of the mealybugs of the world (Insecta: Homoptera: Coccoidea: Pseudococcidae and Putoidae) with data on geographical distribution, host plants, biology and economic importance. Intercept Ltd, Andover, p 686

García Morales M, Denno BD, Miller DR, Miller GL, Ben-Dov Y, Hardy NB (2016) ScaleNet: a literature-based model of scale insect biology and systematics. Database. https://doi.org/10.1093/database/bav118

Hambleton EJ (1976) A revision of the new world mealybugs of the genus Rhizoecus (Homoptera: Pseudococcidae). U S Dep Agric Tech Bull 1522:1-88

Hodgson CJ (2012) Comparison of the morphology of the adult males of the rhizoecine, phenacoccine and pseudococcine mealybugs (Hemiptera: Sternorrhyncha: Coccoidea), with the recognition of the family Rhizoecidae Williams. Zootaxa 3291:1-79

Jansen MGM (2001) An annotated list of the scale insects (Hemiptera: Coccoidea) of the Netherlands. Entomologica 33:197-206

Jansen MGM (2003) A new species of Rhizoecus Kunkel d'Herculais (Hemiptera, Coccoidea, Pseudococcidae) on bonsai trees. Tijdschr Entomol 146:297-300 
Kaydan MB, Konczné Benedicy Z, Kiss B, Szita É (2016) A survey of scale insects in soil samples from Europe (Hemiptera, Coccomorpha). ZooKeys 565:1-28. https://doi.org/10.3897/zookeys.565.6877

Kaydan BM, Konczné Benedicty Z, Kondo T, Ramos-Portilla AA, Szita É (2018) The genus Coccidella Hambleton (Hemiptera: Rhizoecidae) with description of two new species. Neotrop Entomol 47:369-379. https://doi.org/10.1007/s13744-017-0566-3

Kosztarab M, Kozár F (1988) Scale insects of Central Europe. Akadémiai Kiadó, Budapest, p 456

Kozár F, Foldi I (2004) Description of new genera and species in the tribe Rhizoecini (Homoptera, Coccoidea, Pseudococcidae). Acta Zool Acad Sci Hung 50:153-182

Kozár F, Konczné Benedicty Z (2002) Description of the Marottarhizoecus issisi gen. et sp. nova (Homoptera, Coccoidea, Pseudococcidae, Rhizoecinae) from Africa with a review and key of the subfamily. Boll Zool Agr Bachic Ser II 34:213-218

Kozár F, Konczné Benedicty Z (2003) Description of four new species from Australian, Austro-oriental, New Zealand and South Pacific regions (Homoptera, Coccoidea, Pseudococcidae, Rhizoecinae), with a review, and a key to the species Ripersiella. Boll Zool Agr Bachic Ser II 35:225-239

Kozár F, Konczné Benedicty Z (2005) A new genus and species from Rhizoecinae (Homoptera Coccoidea Pseudococcidae), with a phylogeny and key for the genera. Boll Zool Agr Bachic Ser II 37:141-150

Kozár F, Konczné Benedicty Z (2007) Rhizoecinae of the world. Plant Protection Institute, Hungarian Academy of Sciences, Budapest, p 617

Matile-Ferrero D (1976) La faune terrestre de l'lle de Sainte-Helene. 7. Coccoidea. Musee Royal de l'Afrique centrale, Tervuren, Belgique, Annales (Serie 8). Sci Zool 215:292-318
Ramos-Portilla AA (2014) Second time synonymy of Rhizoecus perprocerus De Lotto, 1961, with Rhizoecus graminis (Hambleton, 1946) (Hemiptera: Rhizocidae). Bol Mus Entomol Univ Valle 15:7-11

Ramos-Portilla AA, Caballero A (2016) Rhizoecus colombiensis Ramos \& Caballero, a new species of hypogeal mealybug (Hemiptera: Coccomorpha: Rhizoecidae) and a key to the species of Rhizoecus from Colombia. Zootaxa 4092:55-68. https://doi.org/10.11646/ zootaxa.4092.1.3

Southwood TRE, Henderson PA (2000) Ecological methods, 3rd edn. Blackwell Science, Oxford, p 593

Tanaka H (2016) A new genus and species of Rhizoecidae (Hemiptera, Sternorryncha, Coccomorpha) associated with Acropyga yaeyamensis (Hymenoptera, Formicidae, Formicinae). ZooKeys 616:115-124

Tang FT (1992) The Pseudococcidae of China. Shanxi Agricultural University, Taigu, $\mathrm{p} 768$

Williams DJ (1973) Two cactus-feeding mealybugs from Argentina (Homoptera, Coccoidea, Pseudococcidae). Bull Entomol Res 62:565570

Williams DJ (1998) Mealybugs of the genera Eumyrmococcus Silvestri and Xenococcus Silvestri associated with the ant genus Acropyga Roger and a review of the subfamily Rhizoecinae (Hemiptera, Coccoidea, Pseudococcidae). Bull Nat Hist Mus Entomol 67:1-64

Williams DJ (2004) Mealybugs of Southern Asia. The Natural History Museum, Kuala Lumpur: Southdene SDN. BHD, p 896

Williams DJ, Granara de Willink MC (1992) Mealybugs of Central and South America. CAB International, Wallingford, p 635

Publisher's Note Springer Nature remains neutral with regard to jurisdictional claims in published maps and institutional affiliations. 